\title{
Reducing The Number Of Flips In Trilateration With Noisy Range Measurements.
}

\author{
Hüseyin Akcan and Cem Evrendilek \\ Izmir University of Economics, Balçova, Izmir, Turkey, 35330. \\ E-mail: \{huseyin.akcan, cem.evrendilek\}@ieu.edu.tr
}

\begin{abstract}
Many applications in wireless networks depend on accurate localization services to operate properly. Trilateration is a widely used range-based localization method that can operate in polynomial time, given that the distance measurements are precise. However in real-world, range measurements tend to have errors due to internal and external factors. Flip ambiguities that occur during trilateration as a consequence of imprecise range measurements turn localization via trilateration into an intractable problem. In this paper, we analyze flip ambiguities due to range measurement errors and propose a heuristic solution that tries to minimize the number of flips in trilateration even in highly noisy environments. We simulate our algorithms under various noise scenarios and observe that the use of our heuristic based solution effectively decreases the number of flips in trilateration and increases the accuracy of the localization.
\end{abstract}

\section{Categories and Subject Descriptors}

C.2.1 [Network Architecture and Design]: Wireless Communications

\section{General Terms}

Algorithms

\section{Keywords}

trilateration, localization, NP-hardness, wireless sensor networks.

\section{INTRODUCTION}

Recently, advances in technology have made it possible to manufacture sensor nodes equipped with microprocessors, memory, and radios for wireless communication. Researchers have, as a consequence, started to study a new type of network structure, namely wireless sensor networks, formed by a cluster of these sensor nodes. Wireless sensor networks having a wide spectrum of application areas

*This work is supported by TUBITAK Career Grand no: $112 \mathrm{E} 099$.

Permission to make digital or hard copies of all or part of this work for personal or classroom use is granted without fee provided that copies are not made or distributed for profit or commercial advantage and that copies bear this notice and the full citation on the first page. To copy otherwise, to republish, to post on servers or to redistribute to lists, requires prior specific permission and/or a fee.

MobiDE'13, June 23, 2013, New York, New York, USA.

Copyright 2013 ACM 978-1-4503-2197-6/13/06 ...\$15.00. in daily life need some basic services for efficient operation. One such basic requirement is localization.

Localization can be used to specify the three dimensional absolute position of any sensor on earth as well as its relative position with respect to one or more reference nodes in a network. In order to obtain absolute position data, satellite based localization (e.g. GPS) is used. Global positioning systems are based on the availability of satellites revolving around the world and require a clear sight of at least four satellites in order to work. GPS based localization in wireless sensor networks in general suffer from some major drawbacks. First of these, is the increased hardware cost and energy dissipation incurred by placing a GPS device on each sensor. Additionally, the requirement for a clear sight of satellites is not admissible especially indoors, in places with tall buildings, or in geographically obstructed outdoor areas such as forests. Another drawback is the imprecision of GPS based localization when nodes stay close to each other. If the nodes are closely clustered in a small area, GPS based localization schemes do not provide enough accuracy. Today, low cost GPS devices used in routine applications other than military have typically an average measurement error of 30 meters [7]. Many applications depend on a level of accuracy for localization that global positioning systems cannot provide [7] [19]. A wireless sensor network where nodes are at a distance of 30 meters or less is one such example. As a result, in localization applications where sensors are in close proximity of each other, range-based alternatives are used.

In range-based localization [1] [2] [3] [10] [21] [23] [26] [29] [36], sensor nodes measure, by well known ranging methods (TOA, TDOA [28] AOA [25], and UWB ranging [16] [30]), the distances to their neighbors. These measurements give us an Euclidean graph where the nodes correspond to sensors and the edges between nodes are affiliated with the measured distances. Range-based localization has its own difficulties, as using only ranging turns the problem into a graph realization problem shown to be NP-complete [33] [35]. Different techniques have been proposed to solve restricted forms of the localization problem in polynomial time. The most widely used among these is trilateration. Trilateration in $2 D$ is defined to be the operation whereby nodes starting with a set of three or more seed nodes determine their own positions through triangulation in terms of the positions of those three or more already localized nodes. A polynomial time solution via trilateration [14] is made possible by assuming that the range measurements are exact. However, it is a commonly accepted fact that in real-world, the range measurements are always prone to errors caused by environ- 
mental noise or device failures. In a prior work [15], we have proved that trilateration in the presence of noisy measurements is NP-complete.

A fundamental outcome of this paper is to make the development of robust algorithms to perform trilateration possible by reducing the flip ambiguity when measurements might include errors. In [20] (Eq. 42) it is shown that considering no flip ambiguity, the localization error is bounded by the hop distance to anchors, sensor density and variance of the measurement errors. Therefore, in this paper, we emphasize reducing the flip ambiguity, as solving the flip ambiguity will lead to a bounded localization error as shown in [20].

Our main contributions in this paper are:

- We propose heuristic solutions to the intractable trilateration problem when distance measurements are imprecise.

- Provide mechanisms to reduce the number of flips in trilateration with noisy range measurements.

- Reduce the localization error that occurs due to flip ambiguity.

The rest of the paper is organized as follows. We describe our methods to reduce the flips in Section 2. We present the experimental evaluation in Section 3. We review the state of the art in Section 4, provide a discussion and future work in Section 5 and we present the concluding remarks in Section 6.

\section{THE PROBLEM DEFINITION}

In this section we present the formal definition of the problem and our proposed solution to reduce the number of flips in trilateration when distance measurements are imprecise. In section 2.1 we present a review of the trilateration problem and the intractability result, and in Section 2.2 we present the details of our solution.

\subsection{Review of Trilateration}

In trilateration, the position of a node is calculated by using distance measurements to three other nodes with known positions stated as anchors. Simply, each distance measurement creates a circle around the anchor node with radius set to the distance between the node and the anchor, and position of the node is in the intersection of these three circles, given that the range measurements are exact. The trilaterion process for exact range measurements is presented in Figure 1. In inaccurate range measurements, the intersection of the first two circles gives two alternative positions or areas and the distance to the third anchor node is used to select the final position. Among the alternative positions, the one with the closest distance to the measured distance in general is selected as the final position.

In cases of imprecise distance measurements, if the inaccuracy of the range value is larger than the differences between the distances of the alternative locations, this can lead the algorithm to select the incorrect alternative position as the final answer, which is called the flip ambiguity. Due to its nature, flip ambiguities have the potential to create huge localization errors. The trilateration with measurement errors is presented in Figure 2.

One other problem with the flips is that they cause the trilateration problem to be intractable as shown in [15]. The

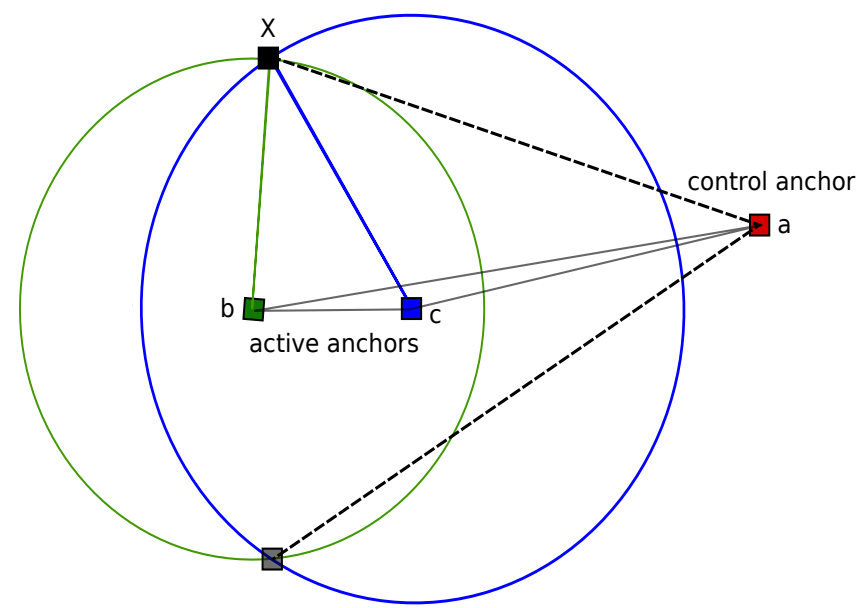

Figure 1: Trilateration with exact range measurements. Active anchors and the control anchor is used to pinpoint the location of the black node.

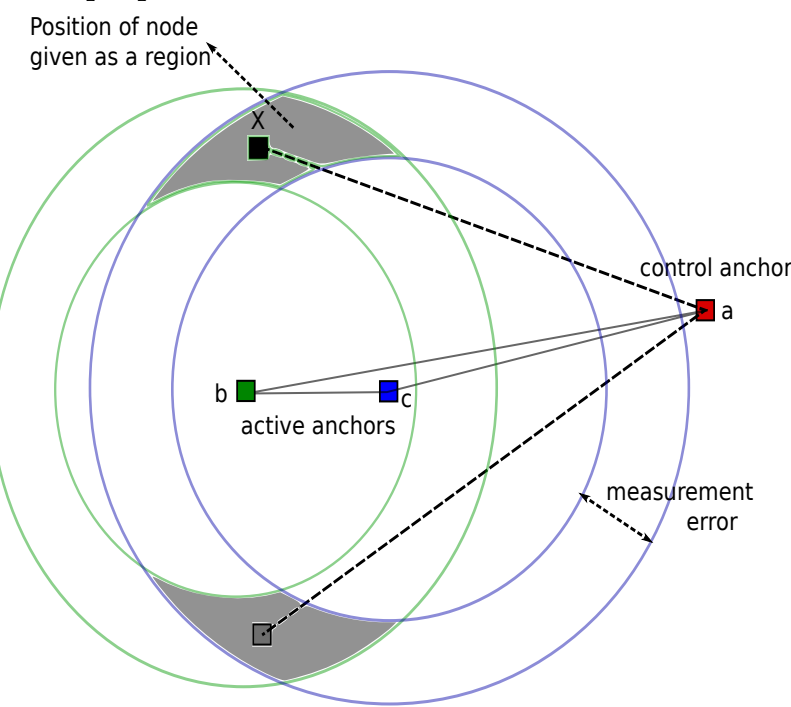

Figure 2: Trilateration with measurement errors. Active anchors are used to calculate the location of the black node in two regions, and the distances to the control anchor is used to finalize the location. Incorrect calculations due to noise can cause a flip in the location of the black node.

definition of the problem, and the theorem proving the intractability result is given here for the sake of completeness.

A point formation [15] $F$ is defined as a realization of nodes with Euclidean coordinates preserving the distances in a graph $G$.

Definition 2.1. [15] Given a trilateration graph, $G(V, E)$, and the maximum constant measurement errors $\epsilon_{i j} \geq 0$ associated with each edge $(i, j) \in E$ where $i, j \in V$, the problem of deciding whether there exists a point formation $F$ where the distances $d_{i j}^{F}$ between any two nodes $i$ and $j$ in $F$ is within $\epsilon$-range of the corresponding distance $d_{i j}^{G}$ in $G$ (i.e. $\left.\left|d_{i j}^{F}-d_{i j}^{G}\right| \leq \epsilon_{i j}\right)$ is called $\epsilon_{i j}-T R I L A T$.

Theorem 2.2. [15] $\epsilon_{i j}-T R I L A T$ is NP-complete.

Examining the proof given in $[15]$ we can conclude that the 
main reason for the intractability result is the flip ambiguity generated due to imprecise distance measurements. Therefore, in this paper, we propose heuristic methods to solve the $\epsilon_{i j}$-TRILAT problem by adding additional constraints to reduce the number of flips during trilateration.

\subsection{Heuristic Solution to Reduce The Number of Flips}

In this section we present our methods to reduce flips due to imprecise range measurements. We define a flip as follows:

Definition 2.3. Given a node $i$ to be localized, and three anchor points $\left\{n_{j} \mid j \in[1 \ldots 3]\right\}$, let us denote the ground truth of the position of $i$ as $P_{i}$ and the calculated position after trilateration as $T_{i}$. If $P_{i}$ and $T_{i}$ are not on the same side of all the three line segments passing through any two of the anchor points, then the position of $i$ is flipped.

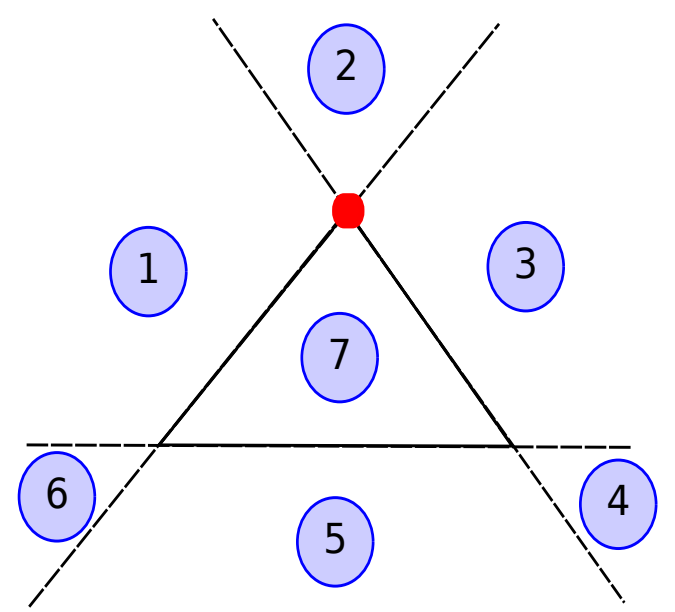

Figure 3: The seven possible regions when trilateration is performed for three anchor nodes.

Figure 3 identify all seven regions in trilateration with three anchors. If a node's real position and the calculated position are not on the same region, the situation will be counted as a flip.

Examining the cases that lead to flip ambiguity, we observe that there exist some safer anchor selections that can avoid the flip ambiguity. Based on this observation, in order to avoid a flip in trilateration with noisy range measurements, we restrict the selection of the trilateration anchors based on various criteria described below. Especially in dense deployments, each node has a certain number of alternative combinations when selecting anchors within communication range. Examining each of these options based on the criteria given in Definition 2.4 below, a node can reduce the probability of a flip, therefore improve the localization accuracy of itself.

In trilateration, as shown in Figure 1, we will call nodes $b$ and $c$ as the active anchors, where the two circles are drawn, and node $a$ will be called as the control anchor, where the nodes will use the distance to node $a$ to resolve the flip ambiguity.

DeFinition 2.4. A safe-triangle $S T$ is defined as the triangle formed by three anchors $\left\{n_{j} \mid j \in[1 \ldots 3]\right\}$, such that the distance of any one anchor to the line passing through the remaining two anchors is larger than a predefined threshold $\gamma$. The $\gamma$ value is also called in our paper as the safety margin.

The threshold value in safe-triangle can be selected based on the measurement error present in the environment. In our experiments, we analyzed two different versions of the threshold value. In the first version we set $\gamma$ to the measurement error $\epsilon$. In the second version, considering that the localization error in trilateration increases at each iteration, we set $\gamma$ to a multiple of $\epsilon * k$ where $k$ is the trilateration order. Trilateration order can be defined as follows:

Definition 2.5. Trilateration order is the shortest hop distance of any node to the closest anchor node on the trilateration graph.

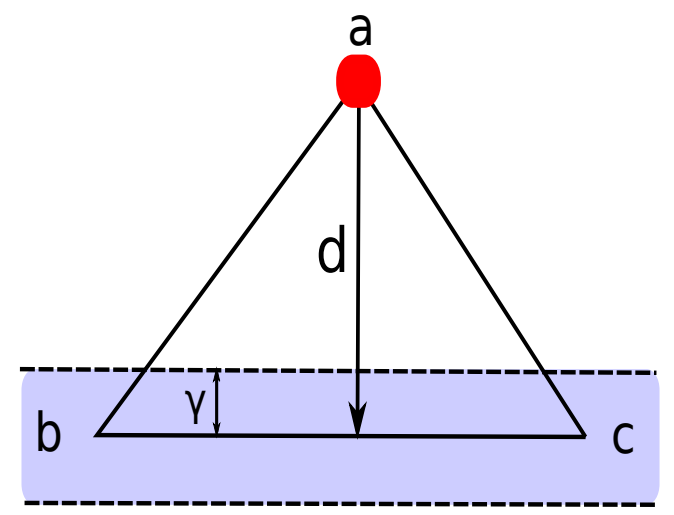

Figure 4: In order to consider the anchors are safe from flips, the distance $d$ of anchor $a$ should be clearly larger than the safety margin $\gamma$.

Due to the nature of trilateration, the process starts from well known anchor points and continue to cover all the nodes in the graph. During trilateration, errors at each iteration tend to accumulate increasing the possibility of flip ambiguity in the later stages of the trilateration. In order to overcome this limitation, we modify safe-triangle to be adaptive, and increase the value of $\gamma$ linearly at each iteration of the trilateration. The advantage of this method is that even though the errors increase at each iteration, the safe-triangle still can check whether the triangle can cause a flip or not. The disadvantage is that since the safety margin increases at each iteration, it gets difficult to find safe-triangles in later stages of the trilateration. This leads to either selecting anchors that are not flip-free, or leaving nodes unlocalized instead of calculating incorrect localization for that node.

The trilateration order gives us the path the trilateration error is propagated from an anchor node to the current node. We follow the trilateration order in $\gamma$ calculations to minimize the effects of the accumulated error throughout the previous rounds of the trilateration. The safe-triangle with a fixed $\gamma$ value is present in Figure 4. Based on Figure 4, in order to avoid a flip, the anchors should be positioned in a way that the $d$ distance from any anchor to the line passing through the remaining anchors should be clearly larger than the $\gamma$ value set as the safety margin.

In our work, we have chosen not to localize nodes that are trilaterated with respect to anchors which does not meet the safe-triangle requirements. The main idea here is that as the trilateration errors tend to accumulate, an erroneous 
localization will potentially introduce flips which have an adverse effect on the later stages of the localization. Therefore, instead of causing an incorrect localization for the node itself and for all future trilaterations, we selected to leave the nodes without safe-triangles unlocalized, and avoid them to interfere with the future trilateration calculations. This effectively decreases the total number of flips as well as the localization error since it minimizes the accumulation of the errors. But the downside is having less number of localized nodes. Overall, we conclude that having less number of nodes more accurately localized is much better than having all nodes localized with huge measurement errors and flip ambiguities.

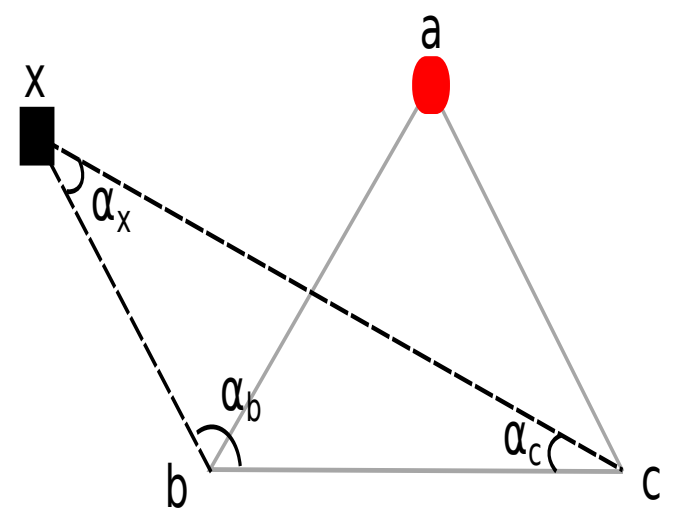

Figure 5: In order to avoid a flip, the distance of node $x$ to the line passing through $b$ and $c$ should be clearly larger than the measurement error. As the position of $x$ is not known, the inner angles are used to enforce this constraint. The inner angles of the triangle formed by nodes $x, b$ and $c$ are required to be larger than a predefined threshold $\alpha$.

Even though safe-triangle requirement solves the possible flip ambiguity caused by almost collinearly located anchors, flips can still occur if the node to be localized is almost collinear with the active anchors, as shown in Figure 5. The method we propose to avoid this case is called the safe-anchors test, and it is actually a generalization of the safe-triangle test to the node to be localized. We give the formal definition of the safe-anchors test below.

DeFinition 2.6. Safe-anchors are defined as the set of anchors $a, b$, and $c$, such that the distance of node $x$ to be localized to the line passing through the active anchors $b$ and $c$ should be larger than the safety-margin.

As shown in Figure 5, if the distance of node $x$ to the line passing from active anchors $b$ and $c$ is less than the safety margin, there is a possibility of flip. In order to avoid the flip, we have to make sure that the distance is larger than the safety margin, for which we require the position of node $x$. As we cannot know the position of $x$ before it is localized, we modify Definition 2.6 to use the angles calculated through distance measurements. Based on this new definition we say that the anchors used in trilateration are safe-anchors if the inner angles of the triangle formed by the position of node $x$ and the positions of the active anchors is larger than a preset angle value $\alpha$. Since all the lines in Figure 5 correspond to range measurements and known, the inner angle values of the triangle can be calculated trivially using law of cosines. We observe in our experiments that the safeanchors method reduces the number of flips further, and for small measurement errors the number of flips even drops to zero in our simulations.

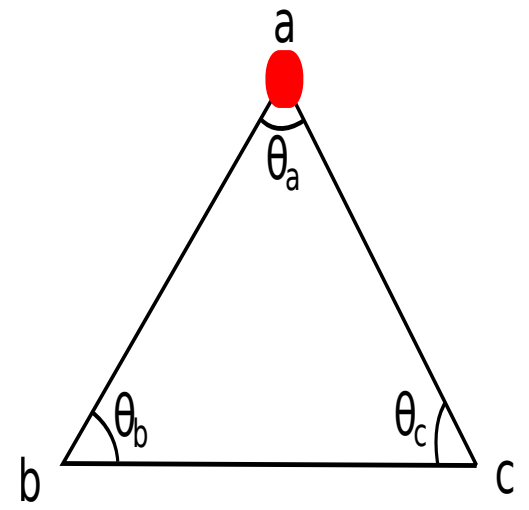

Figure 6: Triangle inner angles test. The inner angles of the triangle formed by the anchors are required to be larger than a predefined threshold $\beta$.

In addition to safe-triangle and safe-anchors requirements, to guarantee that the triangle formed by the three selected anchors is flip free, we use an additional test called triangle inner angle test. In this test, we force the inner angles of the triangle formed by the three anchor nodes to be larger than a preset value $\beta$. Even though the safe-triangle requirement is a relaxed form of this requirement, and properly selected $\gamma$ values entirely cover this test, in experiments, especially in reducing the localization error, we observe practical uses of this more restricted test. Therefore, we present the triangle inner angle test briefly here for the sake of completeness. The triangle inner angle test is shown in Figure 6.

In this section we presented three tests that we use during anchor selection to reduce the number of flips in trilateration with imprecise distance measurements. We evaluate the efficiency of these tests in the experiments section below.

\section{EXPERIMENTS}

In this section, we present the experimental evaluation of the self-triangle tests on the performance of the localization algorithms. We first perform the safe-triangle tests with fixed and increasing safety margins in Section 3.1. Later in Section 3.2 we perform additional experiments to see the effects of the triangle inner angle tests and safe-anchors tests on the localization performance and the total number of flips resulting from the trilateration algorithm.

\subsection{Safe-triangle experiments}

In this section, we perform the safe-triangle tests using two different parameters. As the first parameter, we select the safety margin as a fixed distance value equal to the measurement error, and as the second parameter, we increase the safety margin linearly with the trilateration order again as a multiple of the measurement error. Simulations are performed on a 100 by 100 unit area, with 100 wireless nodes, each with wireless range set to 40 units. Each simulation is performed 1000 times and the average values are reported.

We analyze our results related to the measurement error range mainly in two parts, expected and high measurement 


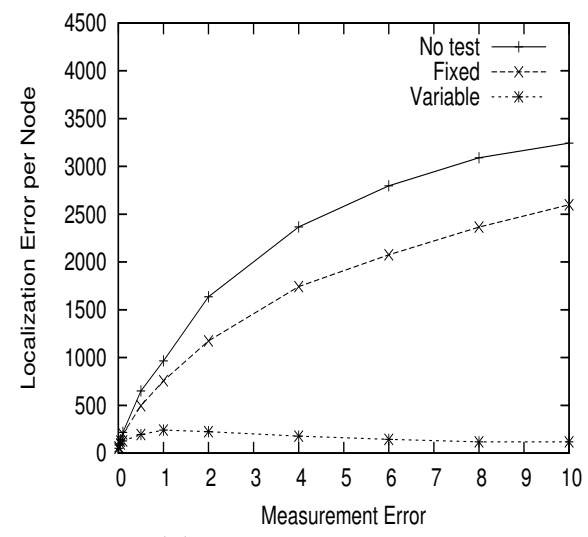

(a) Localization Error

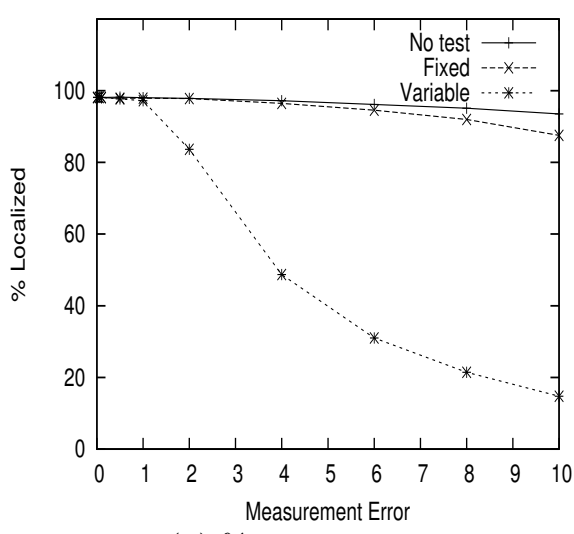

(b) \% Localization

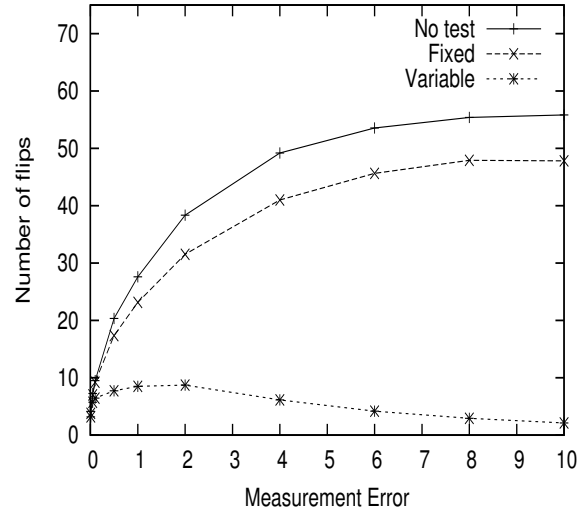

(c) Number of flips

Figure 7: Safe triangle tests comparing the results of when the test is not used, used with fixed safety margin and with variable increasing safety margin.

errors respectively. The division is based on the reported measurement errors for Ultra-wideband radios which is close to $2 \mathrm{~cm}^{1}$ for our simulation conditions. Even though the expected range ( $<2$ units) is our particular interest, we present the simulation results of the high measurement error ranges for the sake of completeness.

The results of the experimentation is presented in Figure 7. Figure 7(a) reports the average localization error per node, where only the localization errors of the localized nodes are included. As we see from the figure, safe-triangle test with variable safety margin decreases the localization error several orders of magnitude.

Figure 7(b) presents the percentage of nodes localized in the same experiment. As we see from the figure, the variable safety margin decreases the number of localized nodes for higher measurement error values, however, as seen again in Figure 7(a), the nodes that are localized are accurately localized. We can argue here that localizing less nodes accurately is better than localizing more nodes in a non accurate way for many application areas.

In Figure $7(\mathrm{c})$ we can see the number of flips occurring if the safe-triangle test is not used, or for the two different safety margins used. As we see, the variable safety margin decreases the number of flips dramatically. The decrease in number of flips is particularly important for us, because as we show in [15] the flips make the localization intractable. One other observation we can make in Figures 7(a) and 7(c) is the correlation between the decrease in the number of flips and the localization error. Therefore, reducing the number of flips has dual benefits for our case.

As a conclusion we can summarize that safe-triangle with variable safety margin option reduces the number of flips and the total localization error in the system, while performing accurate localization.

\subsection{Triangle inner angle and safe-anchors ex- periments}

In this section, we evaluate methods to further reduce the number of flips in trilateration. In order to do so, we perform

\footnotetext{
${ }^{1}$ Time Domain PulsOn P410 Data sheet

http://www.timedomain.com/datasheets/320-0289D P410

Data Sheet.pdf
}

the triangle inner angle test and safe-anchors test to observe their effect on the number of flips.

Figure 8 presents the results of using safe-triangle with variable safety margin option, and in addition using the triangle inner angle test with various inner angle values changing from none to 50 degrees. In Figure 8(a), we can observe that the use of triangle inner angle test reduces the localization error per node. If we cross examine Figure 8(b) at the same time, we can see that again for larger error values ( $>2$ units), the percent of localized nodes drop. The result again tells us that even though less nodes are localized, the accuracy of the localized nodes are higher than not using the triangle inner angle test.

In Figure 8(c) we also observe that the number of flips of the trilateration algorithm further drops by using the triangle inner angle test. From Figure 8, we can conclude that selecting the inner angle as 30 degrees is a reasonable option.

In Figure 9, we present the safe-triangle with variable safety margin option with safe-anchors test for variable angles. The angle values in the safe-anchors tests vary from none to 50 degrees. If we cross examine Figures 9(a) and 9 (b), we can clearly see that the use of safe-anchors tests reduces the localization error. Also for larger measurement errors, percent of nodes localized drops while the localization accuracy stays the same. The result is similar to the result we get from using triangle inner angle test, such that the use of safe-anchors tests increases the accuracy of the localization, and avoid incorrect localization for large measurement errors.

In Figure 9(c), we observe that the use of safe-anchors tests further drops the number of flips in the trilateration process. From Figures 9, we can conclude that selecting the safe-anchors test angle as 20 degrees is a reasonable option.

In order to directly compare the effects of triangle inner angle test and safe-anchors test on the localization performance, we also present the results of applying these two tests together. In Figure 10 we presents the result of this experimentation, where using only one of these tests and using them together are compared, while the safe-triangle test is performed with variable safety margin parameter. Figure 10(a) presents the change in localization error for these various tests. As we see from the figure using the trian- 


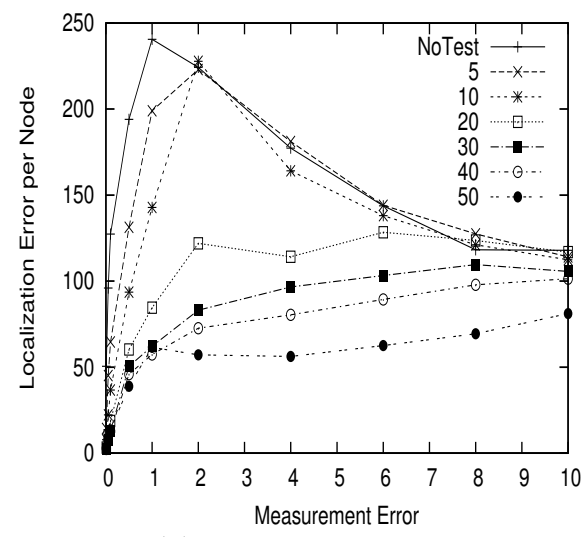

(a) Localization Error

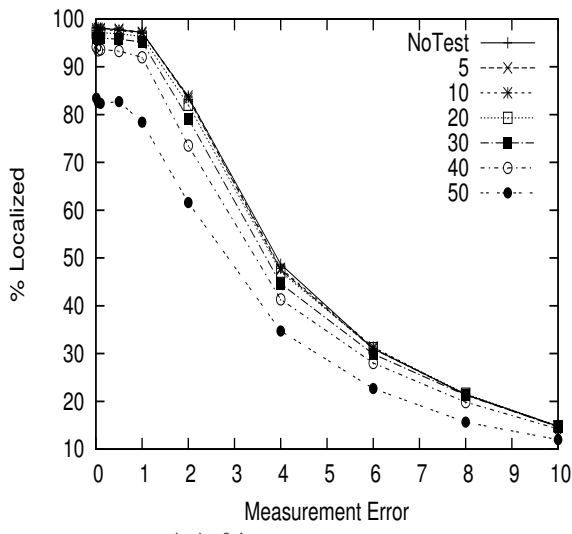

(b) $\%$ Localization

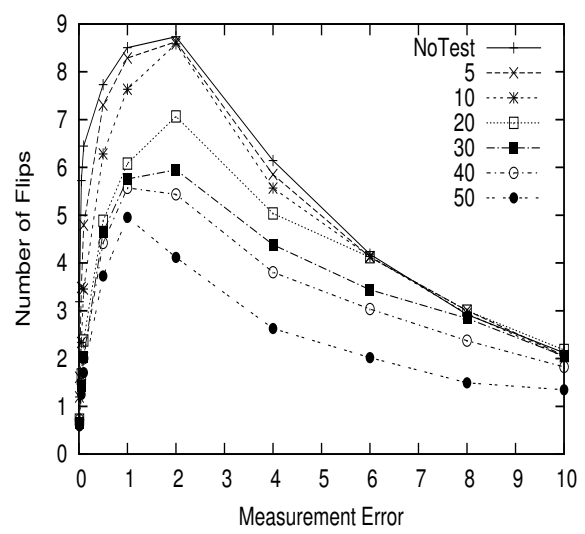

(c) Number of flips

Figure 8: Triangle inner angle tests with various inner angle values.

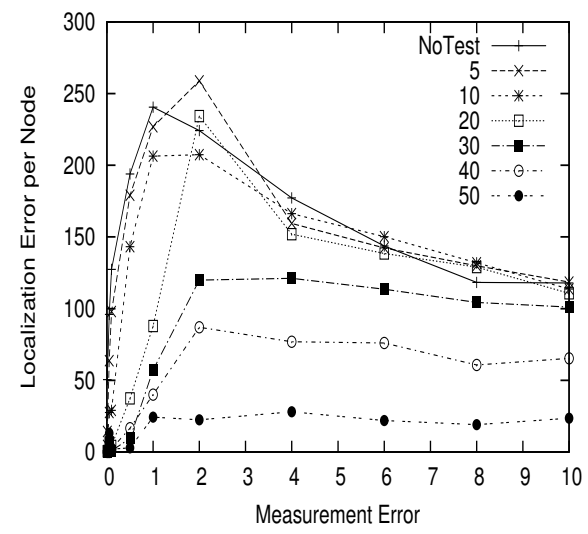

(a) Localization Error

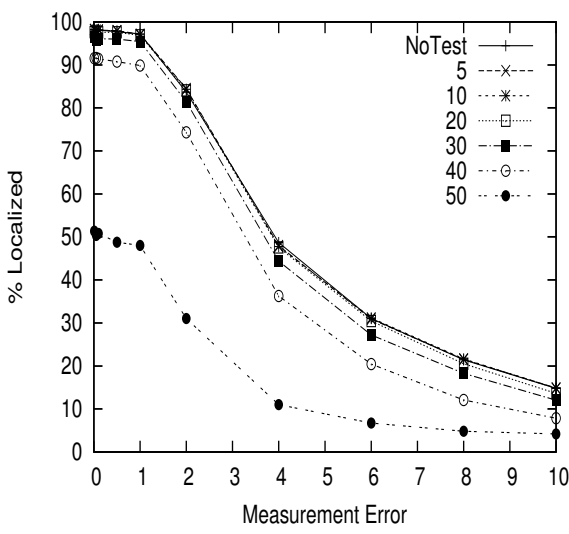

(b) \% Localization

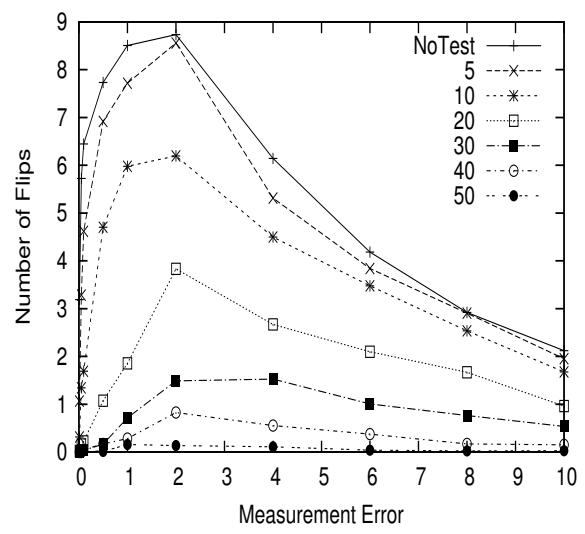

(c) Number of flips

Figure 9: Safe-anchors tests with various angle values.

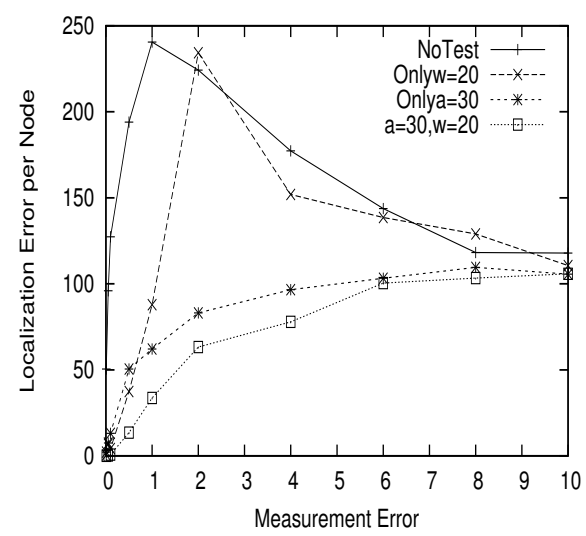

(a) Localization Error

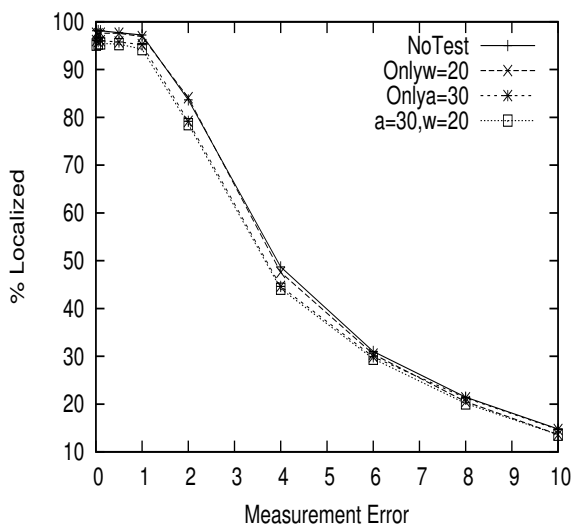

(b) $\%$ Localization

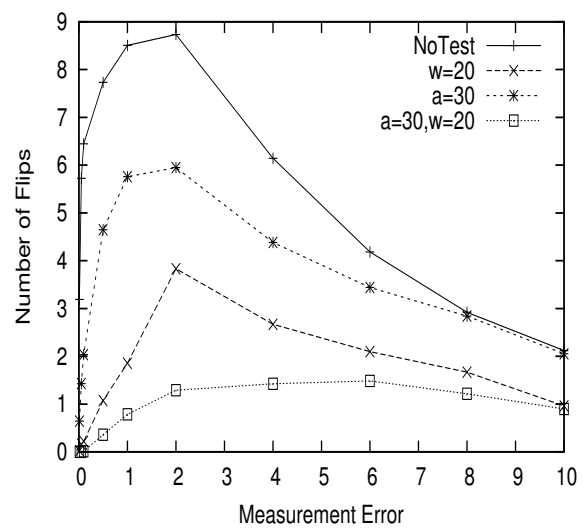

(c) Number of flips

Figure 10: Results of applying the safe triangle tests along with the triangle inner angle tests and safe-anchors tests.

gle inner angle test reduces the localization error more than safe-anchors test but we can achieve better results by using them together.

The percent localization of the nodes for both approaches are similar to each other, as seen in Figure 10(b). In Figure 10 (c) we also observe that safe-anchors test is more successful in reducing the number of flips compared to triangle inner angle test. However, using both tests together is still 
superior to each single approach alone. We can also observe that the number of flips is practically zero for expected real world measurement errors.

From the experimentation described above, we can summarize that the use of safe-triangle test achieves better results, both by performing accurate localization and reducing the number of flips. The number of flips can further be reduced by performing additional tests. Within these tests, the triangle inner angle test is better when it comes to reduce the localization error, and the safe-anchors test is better in case number of flips is the main concern. However, applying these two tests together is superior to each single approach alone both for localization error and number of flips.

\section{RELATED WORK}

Traditionally localization is done using satellite based systems, such as GPS. One major problem with these satellite systems is that they do not provide the required accuracy for some localization applications [8] [13] [22] [24] [31] [32]. Boukerche et al. [7] classifies applications designed for vehicular ad-hoc networks based on their localization accuracy requirements as low, medium and high accuracy applications. Based on these requirements, low accuracy applications can tolerate localization errors between 10 and 30 meters, medium accuracy applications work with sub-meter localization errors and high accuracy applications require at most sub-feet localization errors. Example applications such as geographical routing, data dissemination, and map localization are considered as low accuracy applications. Medium accuracy applications are given as cooperative cruise control, cooperative intersection safety and blind crossing applications. Examples for high accuracy applications are given as vision enhancement systems, collision warning systems and automatic parking systems.

Range based localization [1] [2] [3] [10] [21] [23] [26] [29] is successfully used in applications that global positioning systems fail to provide the required accuracy, such as when the nodes are within short range to each other, or located indoors where the global positioning systems do not work. Range based localization is based on direct measurement of distances among peers in wireless networks [17] [27] [28]. The distances can be presumed to be known apriori for static nodes, or can be measured dynamically by using well known ranging methods such as TOA, TDOA [28], and UWB [16] [30] ranging.

The main difficulty in range based localization is that if only range values are known, the problem reduces to graph realization problem [14] which is known to be NP-hard for general graphs [33] [35]. The problem is still NP-hard [5] [6] for unit disk graphs [11] and globally rigid graphs [12] [14].

A widely used relaxation of the localization problem is trilateration, which is defined in [14] to be the operation whereby a node with known distances to three other nodes which are not collinearly located, determines its own position in terms of the positions of those three already localized nodes. A Trilateration graph in plane or 2D is defined in [14] to be a graph containing as seed the complete graph with three nodes and an ordering on the rest of the nodes such that each node has three edges to the nodes earlier in the sequence. Those who would like a more formal treatment of the rigidity theory are referred to [12] and [14] for the theoretical foundations of network localization in rigidity theory. Assuming that the network graph is a trilateration graph and the range measurements are exact, the trilateration algorithm solves the localization problem in polynomial time.

Even though trilateration solves the localization problem when there are no measurement errors, in real world it is well known that measurements have errors due to internal or external factors. There is already an ongoing research in developing localization algorithms in noisy environments [4] [9] [18] [22] [23] [31] [34]. However, Evrendilek and Akcan [15] recently showed that trilateration with range measurement errors is an NP-complete problem. Moreover, [15] also proves that the problem is intractable even for small measurement errors. Our objective in this paper is to identify the situations that lead the localization problem to be intractable, and find practical solutions that can avoid the NP-complete nature of the localization problem.

The main novelty of our work is the identification of the flip ambiguity as the main cause of the localization problem in trilateration. Although the inner angles of the anchor triangle has been examined before [23], our approach is the first to give an exact definition for a flip and reduce the possible number of flips in trilateration with measurement errors. Our approach is the first heuristic solution proposed after trilateration with measurement errors problem is categorized as intractable.

\section{DISCUSSION AND FUTURE WORK}

In this paper, we propose heuristic methods to trilateration with measurement errors problem, shown to be NPcomplete in [15]. The main point of the intractability result in [15] is that flip ambiguities will occur and the existence of the flips turns a mere polynomial time trilateration algorithm to an intractable problem. Therefore, the heuristics we propose in this paper are based on identifying the possible causes of the flip ambiguity, and eliminate the possible flips by a careful selection of the anchors to trilaterate. Based on our work, we identified that the majority of the flips are happening due to close to collinear alignment of the anchor nodes. Adding the measurement errors to the close to collinear anchor alignments causes the control anchor to miscalculate the correct position of an element and then a flip happens. Even though the exact occurrence of the flip depends on the random error at that particular measurement and how close the control anchor is positioned to the active anchors, using a large enough safety margin in the safe-triangle test is sufficient to eliminate most of the flips. In order to further reduce the number of flips, we also proposed the safe-anchors test and the triangle inner angle tests. Even though the inner angles of the triangle formed by the anchors have been examined before [23], the use of a method similar to safe-triangle and safe-anchors is novel in trilateration research.

Although our heuristic methods effectively reduce the flips in trilateration, there is still work needed to prove whether the elimination of the flips is the sufficient and necessary condition to make the trilateration with imprecise measurements problem tractable.

Even though there are methods proposed to achieve accurate localization or trilateration, with noisy range measurements, [15] is the first approach to formalize the intractable nature of the problem. The proof in [15] also gives important feedback on the cause of the intractability, which is identified as the flips. More research is needed in this respect in order to develop trilateration algorithms that are 
also needed to run in real-world, as the real-world always have measurement errors. Therefore, the existence of new localization frameworks that are guaranteed to work in polynomial time even in high noise scenarios is an open problem for the localization community.

\section{CONCLUSION}

In this paper, we propose a heuristic solution in the form of three tests to the intractable trilateration problem when the distance measurements are imprecise. We give a formal definition of the flip occurring on node positions during trilateration and identify the flips as the main source of the intractability result. Therefore, we propose ways to select the anchors during trilateration to reduce the flip ambiguity due to range measurement errors. We also experimentally evaluate our algorithms and show that our proposed methods reduce the number of flips dramatically both for low and high measurement errors.

\section{REFERENCES}

[1] H. Akcan and C. Evrendilek. GPS-free directional localization via dual wireless radios. Computer Communications, 35(9):1151 - 1163, 2012.

[2] H. Akcan, V. Kriakov, H. Brönnimann, and A. Delis. GPS-Free node localization in mobile wireless sensor networks. In Proceedings of the 5th ACM International Workshop on Data Engineering for Wireless and Mobile Access (MobiDE'06), pages 35-42, Chicago, Illinois, USA, June 2006.

[3] H. Akcan, V. Kriakov, H. Brönnimann, and A. Delis. Managing cohort movement of mobile sensors via GPS-free and compass-free node localization. Journal of Parallel and Distributed Computing, 70(7):743-757, 2010.

[4] B. D. O. Anderson, I. Shames, G. Mao, and B. Fidan. Formal theory of noisy sensor network localization. SIAM J. Discrete Math., 24(2):684-698, 2010.

[5] J. Aspnes, T. Eren, D. K. Goldenberg, A. S. Morse, W. Whiteley, Y. R. Yang, B. D. O. Anderson, and P. N. Belhumeur. A theory of network localization. IEEE Transactions on Mobile Computing, 5(12):1663-1678, 2006.

[6] J. Aspnes, D. K. Goldenberg, and Y. R. Yang. On the computational complexity of sensor network localization. In First International Workshop on Algorithmic Aspects of Wireless Sensor Networks (ALGOSENSORS'04), pages 32-44, Turku, Finland, July 2004.

[7] A. Boukerche, H. A. B. F. Oliveira, E. F. Nakamura, and A. A. F. Loureiro. Vehicular ad hoc networks: A new challenge for localization-based systems. Computer Communications, 31(12):2838-2849, 2008.

[8] N. Bulusu, J. Heidemann, and D. Estrin. Gps-less low cost outdoor localization for very small devices. IEEE Personal Communications Magazine, 7(5):28-34, October 2000.

[9] M. Cao, B. D. O. Anderson, and A. S. Morse. Sensor network localization with imprecise distances. Systems \& Control Letters, 55(11):887-893, 2006.

[10] S. C̆apkun, M. Hamdi, and J.-P. Hubaux. GPS-free Positioning in Mobile Ad Hoc Networks. Cluster Computing, 5(2):157-167, 2002

[11] B. N. Clark, C. J. Colbourn, and D. S. Johnson. Unit disk graphs. Discrete Mathematics, 86(1-3):165-177, 1990.

[12] R. Connelly. Generic global rigidity. Discrete \& Computational Geometry, 33(4):549-563, 2005.

[13] L. Doherty, K. Pister, and L. El Ghaoui. Convex position estimation in wireless sensor networks. In INFOCOM, pages 1655-1663, Anchorage, AK, USA, 2001.

[14] T. Eren, O. Goldenberg, W. Whiteley, Y. Yang, A. Morse, B. Anderson, and P. Belhumeur. Rigidity, computation, and randomization in network localization. In INFOCOM, volume 4, pages 2673-2684, Hong Kong, China, March 2004.

[15] C. Evrendilek and H. Akcan. On the complexity of trilateration with noisy range measurements. Communications Letters, IEEE, 15(10):1097 -1099, October 2011.

[16] S. Gezici, Z. Tian, G. Giannakis, H. Kobayashi, A. Molisch, H. Poor, and Z. Şahinoğlu. Localization via ultra-wideband radios: a look at positioning aspects for future sensor networks. Signal Processing Magazine, IEEE, 22(4):70-84, July 2005.

[17] F. Gustafsson and F. Gunnarsson. Mobile positioning using wireless networks. IEEE Signal Processing Magazine, pages 41-53, 2005.

[18] T. He, C. Huang, B. M. Blum, J. A. Stankovic, and T. F. Abdelzaher. Range-free localization and its impact on large scale sensor networks. ACM Trans. Embedded Comput. Syst. 4(4):877-906, 2005

[19] J. Hightower and G. Borriello. Location systems for ubiquitous computing. IEEE Computer, 34(8):57-66, 2001.

[20] B. Huang, C. Yu, and B. Anderson. Understanding error propagation in multi-hop sensor network localization. IEEE Transactions on Industrial Electronics, 10.1109/TIE.2012.2236991, 2012.

[21] R. Iyengar and B. Sikdar. Scalable and distributed GPS free positioning for sensor networks. In Proceedings of the IEEE International Conference on Communications (ICC'03), pages 338-342, Anchorage, AK, USA, May 2003.

[22] J. Liu, Y. Zhang, and F. Zhao. Robust distributed node localization with error management. In Proceedings of the 7th ACM Interational Symposium on Mobile Ad Hoc Networking and Computing (MobiHoc'06), pages 250-261, Florence, Italy, May 2006.

[23] D. Moore, J. Leonard, D. Rus, and S. Teller. Robust distributed network localization with noisy range measurements. In Proceedings of the 2nd International Conference on Embedded Networked Sensor Systems (SenSys'04), pages 50-61, Baltimore, MD, USA, 2004.

[24] R. Nagpal, H. E. Shrobe, and J. Bachrach. Organizing a global coordinate system from local information on an ad hoc sensor network. In Proceedings of the 2nd International Conference on Information Processing In Sensor Networks (IPSN'03), pages 333-348, Palo Alto, California, USA, 2003.

[25] D. Niculescu and B. Nath. Ad hoc positioning system (APS) using AOA. In INFOCOM, volume 3, pages 1734-1743, March 2003.

[26] J. Park, E. D. Demaine, and S. J. Teller. Moving-baseline localization. In Proceedings of the 7th International Conference on Information Processing In Sensor Networks (IPSN'08), pages 15-26, St. Louis, Missouri, USA, April 2008.

[27] N. Patwari, J. N. Ash, S. Kyperountas, A. O. Hero III, R. L. Moses, and N. S. Correal. Locating the nodes: cooperative localization in wireless sensor networks. Signal Processing Magazine, IEEE, 22(4):54-69, July 2005.

[28] M. Porretta, P. Nepa, G. Manara, and F. Giannetti. Location, location, location. IEEE Vehicular Technology Magazine, pages 20-29, 2008.

[29] N. B. Priyantha, H. Balakrishnan, E. Demaine, and S. Teller. Anchor-free distributed localization in sensor networks. In Proceedings of the First International Conference on Embedded Networked Sensor Systems (SenSys'03), pages 340-341, Los Angeles, CA, USA, 2003.

[30] Z. Şahinoğlu, S. Gezici, and I. Güvenç. Ultra-wideband Positioning Systems: Theoretical Limits, Ranging Algorithms, and Protocols. Cambridge University Press, 2008.

[31] C. Savarese, J. M. Rabaey, and K. Langendoen. Robust positioning algorithms for distributed ad-hoc wireless sensor networks. In USENIX, pages 317-327, Monterey, CA, USA, 2002.

[32] A. Savvides, C.-C. Han, and M. B. Strivastava. Dynamic fine-grained localization in ad-hoc networks of sensors. In Proceedings of the 7th annual International Conference on Mobile Computing and Networking (MobiCom'01), pages 166-179, New York, NY, USA, 2001. ACM Press.

[33] J. B. Saxe. Embeddability of weighted graphs in k-space is strongly np-hard. In 17 th Allerton Conf. Commun. Control Comput., pages 480-489, 1979.

[34] R. Sugihara and R. Gupta. Sensor localization with deterministic accuracy guarantee. In INFOCOM, 2011 Proceedings IEEE, pages $1772-1780$, april 2011.

[35] Y. Yemini. Some theoretical aspects of position-location problems. In 20th Annual Symposium on Foundations of Computer Science (FOCS), pages 1-8, San Juan, Puerto Rico, 1979.

[36] Y. Zhang, S. Liu, X. Zhao, and Z. Jia. Theoretic analysis of unique localization for wireless sensor networks. Ad Hoc Networks, 10(3):623 - 634, 2012. 